\title{
Design of a First-order Annular Inverted Pendulum System
}

\author{
Zheng $\mathrm{Fu}^{1,+}$, Rong $\mathrm{Li}^{1}$ \\ ${ }^{1}$ Henan industrial technician college, Henan Province, China
}

\begin{abstract}
In this paper, a ring inverted pendulum system is designed. The rotating arm is driven by a motor to move back and forth. Drive the pendulum rod to do reciprocating motion perpendicular to the plane. By controlling the pendulum bar from the vertical state to the vertical state, the validity of the control algorithm is verified. Experiments show that the system can realize the stability control of inverted pendulum and is used to study the effectiveness of the algorithm.
\end{abstract}

Keywords: inverted pendulum system; control algorithm; stability control

\section{Introduction}

A rotating inverted pendulum is a nonlinear, variable, strongly coupled, and absolutely unstable system ${ }^{[1-}$ ${ }^{4]}$. The inverted pendulum system can effectively verify the feasibility of the control algorithm ${ }^{[5-6]}$. Therefore, we call the inverted pendulum system a bridge of control theory to practice.

In this paper, a rotating inverted pendulum system is designed. The mechanical part includes a rotating arm, a swinging rod, a stepping motor, and an incremental photoelectric sensor. The hardware part is composed of data acquisition module, power supply module, driving circuit and so on. The experimental results show that the designed system can achieve the desired rotation effect and control objectives.

\section{System Principle}

The annular inverted pendulum system collects two rotary encoder data through the data acquisition card. The two rotary encoders are respectively connected to the rotating rod and the swing rod, and the motor is connected to the rotating rod. Therefore, through the computer we can get the angular displacement of the rotating rod and the pendulum already angular velocity, and then calculate the control amount in the control algorithm according to the formula. The amount of control is transmitted by the computer to the servo motor driver through the data line. There are drives that control the motor to achieve control of the entire system. The working principle diagram is shown in Figure 1.

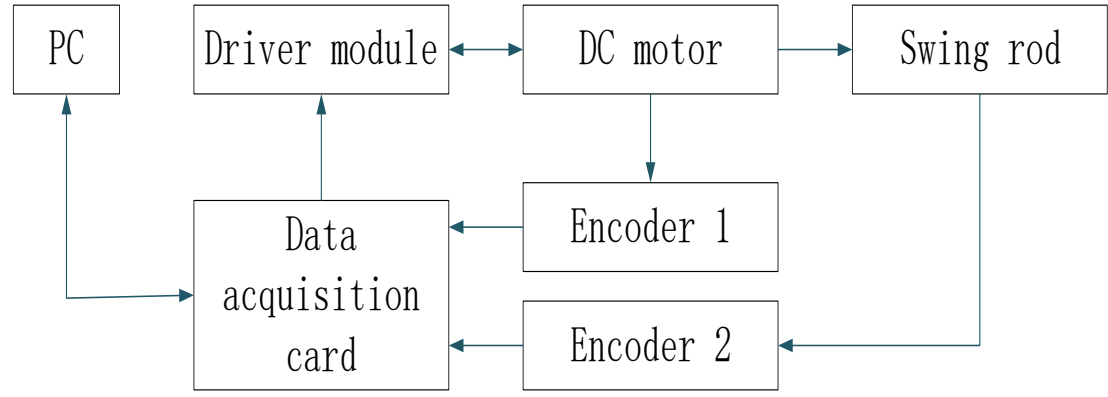

Fig. 1: System schematic block diagram

\footnotetext{
+ Corresponding author. Tel.: +13838362131

E-mail address: 378493652@qq.com
} 


\section{Design of Hardware System}

The inverted pendulum system hardware is mainly composed of a data acquisition module, a power module, a drive circuit, and a motor. Its system block diagram is shown in Figure 2. The system uses the photoelectric encoder to measure the angle of the rotating rod and the swing rod, and transmits the digital signal output by the encoder to the data acquisition module. The data acquisition module processes the transmitted signal and transmits it to the upper computer through the data line, and then Achieve overall control of the system.

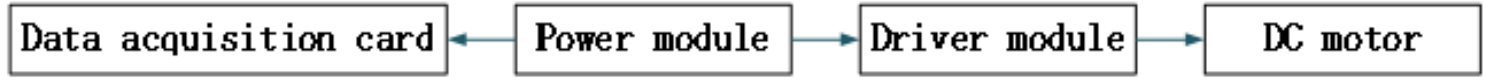

Fig. 2: Hardware system block diagram

\subsection{Stepper motor drive circuit}

Stepper motors are one of the most common control components in industrial control and instrumentation. It has the characteristics that the pulse is proportional to the motor shaft. By the frequency of the pulse signal outputted by the stepping motor drive circuit, the speed of the stepping motor can be changed, and the effect of controlling the rotation angle of the rotating arm can be reached. Its circuit schematic is shown in Figure 3.

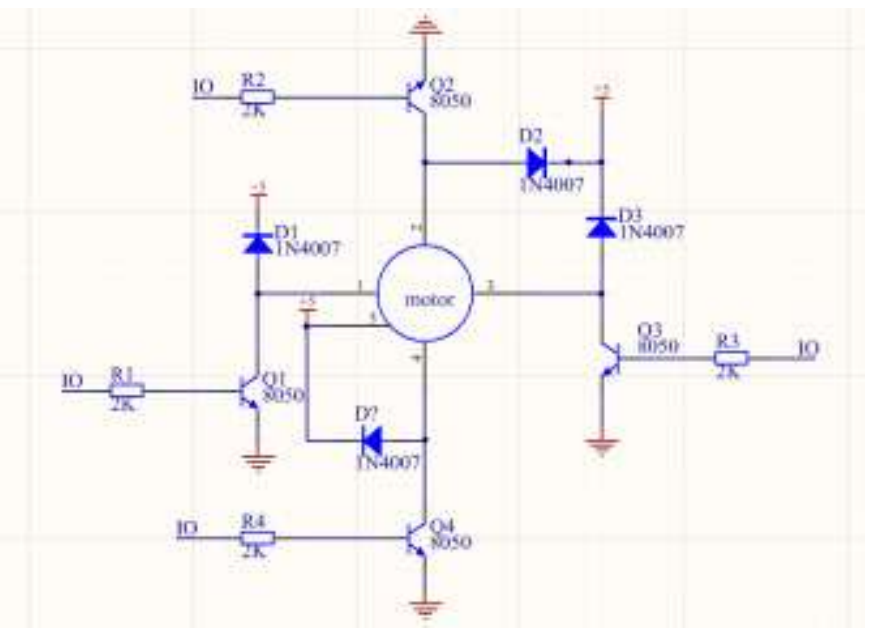

Fig. 3: Stepper motor drive circuit

\subsection{Power module}

The normal operation of the system is inseparable from the power module to provide power to all parts of the system. The modules that the system needs to supply include a data acquisition card, a stepper motor drive circuit, and a rotary encoder. Each electronic module has an operating voltage of $3.3 \mathrm{~V}$. In order to provide a large capacity and output a stable voltage, it can be used for long-term use and can be easily carried, and a lithium battery power supply method is adopted. Its circuit schematic is shown in Figure 4.

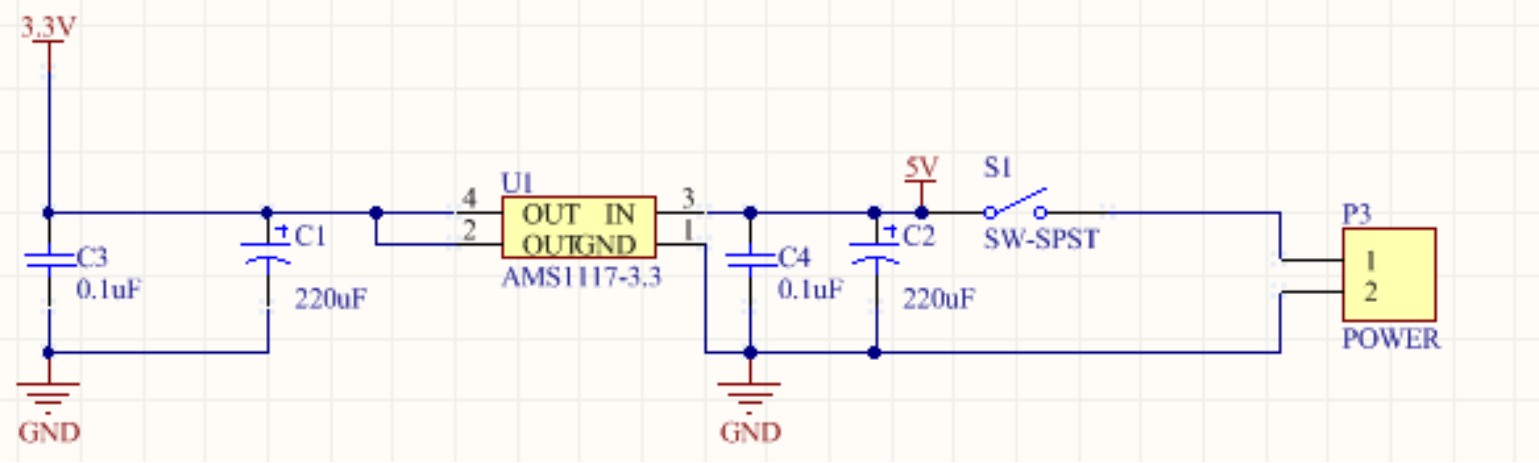

Fig. 4: Circuit schematic diagram of power module 


\section{System Software Design}

The system builds the labview PC software through the system. Implement communication with the data acquisition module, to verify the effectiveness of the system, according to the paper [7], the system is first modeled by the LaGrange method. The mathematical model of the system is obtained by Lagrange method.

$$
\theta=4 *\left(l_{1} \ddot{\theta}_{1} \cos \theta_{2}+\frac{1}{2} l_{2} \dot{\theta}_{1}^{2} \sin \left(2 \theta_{2}\right)+g \sin \theta_{2}\right) /\left(3 * l_{2}\right)
$$

Then by finding the relationship between the control torque exerted on the rotating rod and the energy of the inverted pendulum system, Use the additional control torque to change the energy of the inverted pendulum system until the pendulum is inverted. The structure of its software algorithm is shown in Figure 5.

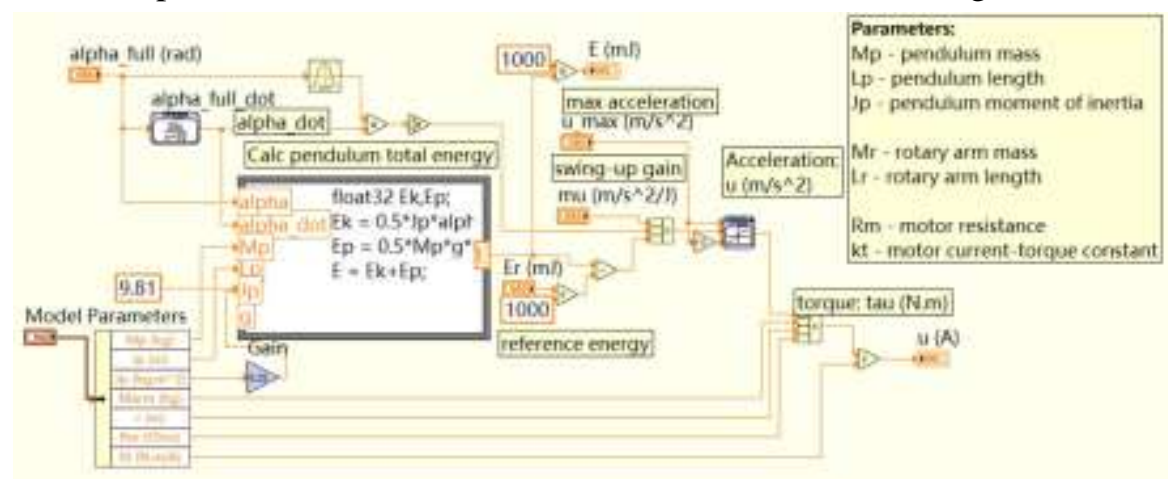

Fig. 5: Structural Chart of Software Algorithms

The system software is built by labview, and the system parameters are set by reserving editable controls. The parameters of inverted pendulum physical system are input into the software system to maintain the correspondence between the software system and the physical system. The swing-up equation of energy method is written into Simulink control, and then the space coefficient is fine-tuned according to the actual operation parameters of the motor, and then the whole system is built.

Since the energy method can only change the inverted pendulum system from the vertical downward state to the vertical upward state, and can not always maintain the stability of the inverted pendulum system, the LQR steady pendulum control is finally introduced into the system to maintain the stability of the system.

\section{Rresults and Analysis}

In this paper, through the combination of hardware and mechanical parts, the construction of the firstlevel rotating inverted pendulum hardware equipment is completed. In fact, the object map is shown in Figure 6.

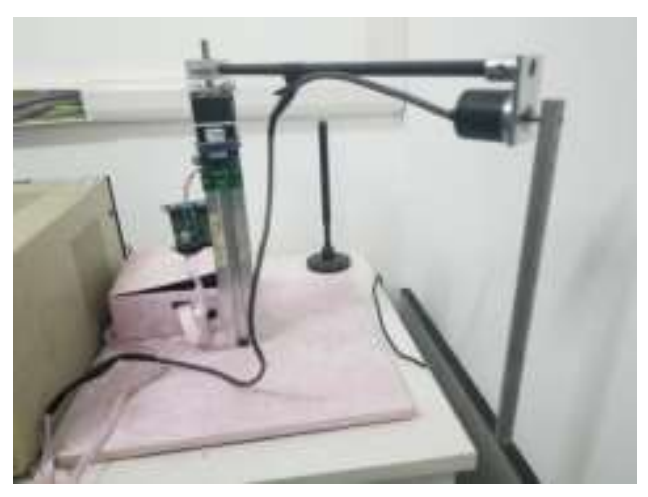

Fig. 6: System Physical Chart

In order to better verify the effectiveness of the hardware system, complete the software construction, read the encoder angle information in real time through the data line to the upper computer software, and use the change of the pulse frequency of the motor drive circuit to realize the control of the rotating rod, and then achieve the pendulum control of the pendulum. In fact, the physical experiment corresponds to the upper computer software interface shown in Figure 7. The interface consists of four parts. The first part is the 
system input channel and sampling frequency system setting module. The module mainly completes the task of system setup. In the process of software construction, several input channels are reserved to ensure that the software system can provide real-time data monitoring for multiple inverted pendulum devices. The second module is the system parameter input module, which is mainly for users to input suitable swing parameters of energy method and LQR stabilization parameters. The third part is the system monitoring module, which can be used to monitor and control the data of swing rod, rotating rod and motor voltage, so as to facilitate users to record the experiment.

As can be seen from Fig. 7, the system completes the swaying motion in about $4.5 \mathrm{~s}$ and keeps it in an upright state. It proves that the system hardware and software design are very complete.

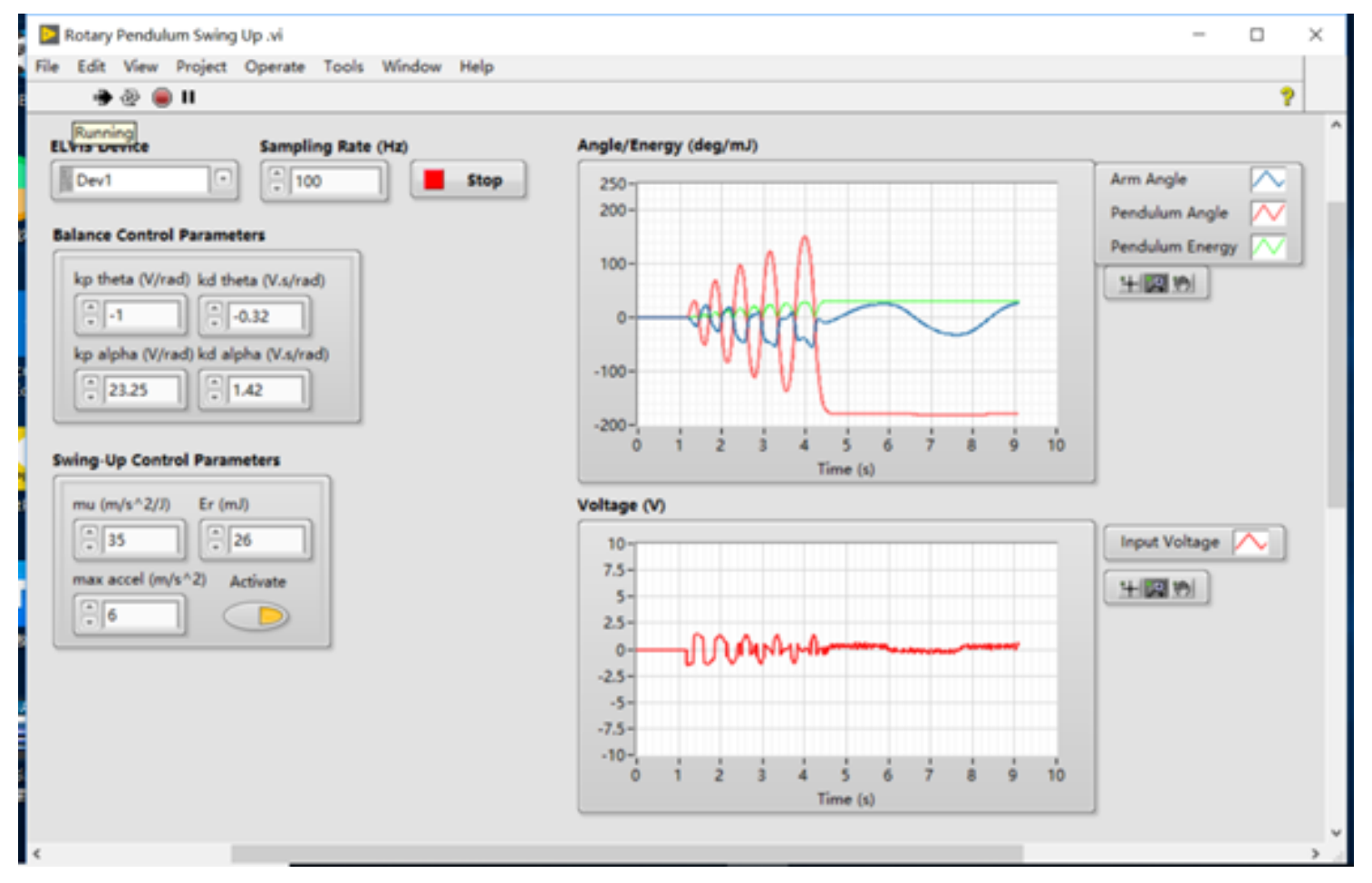

Fig. 7: System Software Test Chart

\section{References}

[1] Limin Du. Nonlinear Controller Design of the Inverted Pendulum System based on Extended State Observer[A].Proceedings of 2015 International Conference on Automation,Mechanical Control and Computational Engineering(AMCCE 2015)[C].Computer Science and Electronic Technology International Society,2015:6.

[2] Jimin Yu. Fuzzy Control of Flexible Double Inverted Pendulum[A]. DEStech Publications,Inc.Proceedings of the 2014 International Conference on Electrical,Control and Automation[C].DEStech Publications, 2014:8.

[3] Aydin,zbey. Nonlinear Control of an Inverted Pendulum on a Cart by a Single Control Law[A]. Information Engineering Research Institute, USA.Proceedings of 2013 International Conference on Intelligent Materials and Mechatronics(IMM 2013)[C].Information Engineering Research Institute,,2013:6.

[4] Chihiro Nakagawa. Steering Performance of an Inverted Pendulum Vehicle with Pedals as a Personal Mobility Vehicle[A]. Shanghai Jiao Tong University.Program and Abstract Book of the sixth Asian Conference on Multibody Dynamics(ACMD 2012)[C].Shanghai Jiao Tong University,2012:1.

[5] Omar Mehrez. ANFIS Based Controller for Double Inverted Pendulum System with Payload[A]. IEEE Beijing Section,China、 Guangdong University of Technology,China.Proceedings of 2011 IEEE International Conference on Intelligent Computing and Intelligent Systems(ICIS 2011) vol.03[C].IEEE Beijing Section,China、Guangdong University of Technology,China:IEEE BEIJING SECTION,2011:5.

[6] Bing Li Department of Automation Huade school of Applied Technology of Harbin Institute of Technology Harbin,China Heming Jia College of Automation Harbin Engineering University Harbin,China. Robust $\mathrm{H} \infty$ Control of The Double-Inverted Pendulum[A]. IEEE.Proceedings of 2011 4th IEEE International Conference on Computer Science and Information 
Technology(ICCSIT 2011) VOL02[C].IEEE:IEEE BEIJING SECTION,2011:5.

[7] ZHANG Ping Department of Electrical and Information Engineering Lanzhou University of Technology Lanzhou,Gansu Province, china HAO Xiaohong and XU Wei Tao Department of Electrical and Information Engineering Lanzhou University of Technology Lanzhou,Gansu Province,china. Dynamic Fuzzy Neural Network based on Lazy Learning algorithm for the Three Stage Inverted Pendulum[A]. Intelligent Information Technology Application Research Association, Hong Kong、 Huazhong Normal University, China、Huazhong University of Science and Technology, China.Proceedings of 2010 Second Asia-Pacific Conference on Information Processing (APCIP 2010)[C].Intelligent Information Technology Application Research Association, Hong Kong、 Huazhong Normal University, China、Huazhong University of Science and Technology,2010:4. 